\title{
Comparison of high-intensity interval versus low-intensity continuous training for myelin synthesis related genes in C57BL/6 mice
}

Maryam Naghibzadeh ${ }^{1 *}$, Rouhollah Ranjbar ${ }^{2}$, Mohammad Reza Tabandeh $^{3}$, Abdolhamid Habibi $^{2}$, Zohreh Ghotbeddin ${ }^{4}$

1. Department of Physical Education and Sports Science, Faculty of Literature and Humanities, Ilam University, Ilam, Iran

2. Department of Exercise Physiology, Faculty of Sport Sciences, Shahid Chamran University of Ahvaz, Ahvaz, Iran

3. Department of Biochemistry and Molecular Biology, Faculty of Veterinary Medicine, Shahid Chamran University of Ahvaz, Ahvaz, Iran

4. Department of Physiology, Faculty of Veterinary Medicine, Shahid Chamran University of Ahvaz, Ahvaz, Iran

*Corresponding author:Tel: +98 9183428195 Fax: +98 8433622915

Address: Department of Physical Education and Sports Science, Faculty of Literature and Humanities, Ilam

University, Ilam, Iran

E-mail: naghibzadehmaryam@yahoo.com

Received; 2018/03/12 revised; 2018/05/25 accepted; 2018/07/19

\section{Abstract}

Introduction: By increasing the scientific focus on myelination, identifying factors that influence the myelination is an important goal for brain health. There are some studies that regular exercise improves myelin sheath and neuronal regeneration. However, the effects of exercise intensities on the myelination remain unclear. Therefore, the purpose of this study was to compare the effect of high-intensity interval (HIIT) versus low-intensity continuous training (LICT) on myelin synthesis-related genes in hippocampus of C57BL/6 mice.

Materials and methods: Male C57BL/6 mice $(\mathrm{n}=30)$ were randomly assigned to 3 groups: control (C), Interval training (IT), and Continuous training (CT). Training programs on the treadmill were performed for 8 weeks and then, the hippocampus of animals was analyzed for the expression of myelin basic protein (MBP) and proteolipid protein (PLP) genes. Data were analyzed using ANOVA.

Results: The result showed that HIIT program significantly increased the mRNA levels of MBP and PLP in comparison with LICT and Control groups $(\mathrm{P}<0.05)$, while no significant differences were observed among the LICT and Control groups $(\mathrm{P}>0.05)$.

Conclusion: Our results showed that HIIT had a more efficient by improving the expression of MBP and PLP genes compared to LICT in the hippocampus.

Keywords: Interval training, Continuous training, Myelin basic protein, Proteolipid protein, Hippocampus

\section{Introduction}

The main functions of myelin sheath are to increase the speed of nerve conduction and protect against axonal degeneration. Myelin comprises between $70 \%$ and $85 \%$ lipids and between $15 \%$ and $30 \%$ proteins.
Myelin basic protein (MBP) and proteolipid protein (PLP) makes $60-80 \%$ of the total proteins myelin $(1,2)$.

The presence of demyelination and the extent of damage to the axons can also

Copyright (C) 2018 Journal of Basic Research in Medical Science. This is an open access article distributed under the terms of the Creative Commons Attribution 4.0 International License (https://creativecommons.org/licenses/by/4.0/) which permits copy and redistribute the material, in any medium or format, provided the original work is properly cited. 
cause mental and physical disorders, muscle weakness, fatigue, impairment of cognition, visual problems, speech disorders (3). There has been growing interest in the study of the effect of exercise training as a health promoting behavior on disease progression in people with demyelination (4). It has been suggested that exercise promote neuroprotection by improving neuronal survival, proliferation, and maturation against demyelination (5).

Recent studies have shown that exercise training was significantly increased PLP expression and reduced demyelination in the exercised group compared with control in brain animals (6). In addition, a study by Kim and colleagues suggested that treadmill walking exercise is effective for the expression of MBP in the corpus callosum (7).

Research in the past decade suggests that high-intensity interval training (HIIT) is more efficient to improve neurotrophic factors and is preferred in clinical populations due to its superior physiological adaptations compared to low-intensity continuous training $(8,9)$. In addition, HIIT programmes are time efficient approach and effects on cognitive function in people with demyelinating diseases of the CNS (10). However, more information on the pathophysiological effects of HIIT is needed to identify the specific mechanisms involved. Previous studies have shown the beneficial effects of HIIT on brain damage in animal models (11). Moreover, the effect of HIIT on myelinating of the CNS is not yet known and more research is needed. Hence, the aim of the present study was to compare the effect of high-intensity interval (HIIT) versus low-intensity continuous training (LICT) on myelin synthesis related genes in hippocampus of C57BL/6 mice.

\section{Materials and methods}

Animals and procedures: The present study was experimental study, Meeddale C57BL/6 mice were purchased from the center for laboratory animals of the Faculty of Veterinary Medicine of Shahid Chamran University of Ahvaz, Iran. The mice ( $\mathrm{n}=10$ per group) were divided randomly into 3 groups: 1. Control (C), 2. Interval training (IT), 3. Continuous training (CT). Animals housed in a temperature-controlled room $\left(23 \pm 2{ }^{\circ} \mathrm{C}\right)$ under a 12-h light/dark cycle (lights on at 07:00 hours), with free access to food and water.

Exercise training protocols: Exercise training was performed on a 5-lane treadmill (Danesh Salar, Iran) at 5-10 $\mathrm{m} / \mathrm{min}, 0 \%$ grade, for $5-10 \mathrm{~min} /$ day for 1 week to ease their adaptation to the new environment, and the MAX test was performed to access the maximal exercise capacity. Exercise training was performed on the basis of overload principle 5 days/week for 8 weeks. The time and intensity increased every two weeks for the CT and HIIT groups. Warm-up and cooldown were performed for $3 \mathrm{~min}$ at $50 \%$ of maximal exercise capacity. Intensity of CT group correspond to $75 \%$ of maximal exercise capacity. In the IT group, each interval was performed for 2-minute at an intensity of $90 \%$ of maximal exercise capacity, followed by active rest that was performed between intervals for $60 \mathrm{~s}$ at $50 \%$ of maximal exercise capacity. In the control group, mice were left on the treadmill, without running, for the same period as the exercise groups.

Tissue preparation: Animals were decapitated under deep anesthesia (Ketamine, 60-80 mg/kg and Xylazine, 8 $\mathrm{mg} / \mathrm{kg}$; IP) $48 \mathrm{~h}$ after the last exercise session and hippocampus and corpus callosum were immediately removed and stored at $-80{ }^{\circ} \mathrm{C}$.

Gene expression analysis of MBP and PLP: Real time PCR was performed using YTA qPCR ${ }^{\mathrm{TM}}$ Master Kit for SYBR Green $I^{\circledR}$ (Yekta Tajhiz, Iran) on a Lightcycler ${ }^{\circledR}$ Detection System (Roche, USA). Total RNA from cells was isolated using the $\mathrm{RNX}^{\mathrm{TM}}$ Reagent (SinaClon Bioscience, Iran) following manufacturer's protocol. 
Concentration of extracted RNA was calculated at a wavelength of $260 \mathrm{~nm}$ using Nanodrop spectrophotometry (Eppendorf, Germany). To detect the purity of RNA, its optical density (OD) absorption ratio 260/280nm was determined and sample having a ratio > 1.8 were used for cDNA synthesis. The primers (Bioneer, South Korea) used in study are shown in Table 1.

Table 1. Characteristics of primers used in the present study.

\begin{tabular}{lc}
\hline Gene & Sequence \\
\hline MBP-F & AGTCGCAGAGGACCCAAGAT \\
MBP-R & GACAGGCCTCTCCCCTTTC \\
PLP-F & CACTTACAGCAGGTGATTAGAGG \\
PLP-R & AAACAAGAGATAAACAACTGGGA \\
GAPDH-F & CTGGAGAAACCTGCCAAGTA \\
GAPDH-R & GAAGAGTGGGAGTTGCTGTT \\
\hline
\end{tabular}

$\mathrm{F}$, forward; $\mathrm{R}$, reverse

Reactions were prepared in a12.5 $\mu \mathrm{l}$ mixture containing $6.25 \mu \mathrm{l} \mathrm{qPCR}^{\mathrm{TM}}$ Master Kit for SYBR Green $I^{\circledR}$ (Yekta Tajhiz, Iran), $0.25 \mu$ of each primer $(200 \mathrm{nM} / \mathrm{L}), 3$ $\mu \mathrm{l}$ cDNA (100 ng), and $2.75 \mu \mathrm{l}$ nuclease. The Real time PCR protocol were performed with the following settings: 5 min denaturation at $94^{\circ} \mathrm{C}$ followed by 45 cycles of $94^{\circ} \mathrm{C}$ for $15 \mathrm{sec}$, and $60^{\circ} \mathrm{C}$ for 30 sec. Reactions were performed in triplicate. Two separate reactions without cDNA or with RNA were performed in parallel as controls. GAPDH was used as the internal control in a real time PCR instrument, and data was analyzed using $2^{-}$ $\Delta \Delta \mathrm{Ct}$ method and using Lightcycler $96^{\circledR}$ software. Validation of assay was carried out to check that the primer for the calibrator (GAPDH) and target genes had similar amplification efficiencies. All qPCR analysis was performed according to The Minimum Information for Publication of Quantitative Real-Time PCR Experiments (MIQE) guideline.

\section{Statistical analysis}

Results were analyzed in SPSS software (version 21.0) and presented as means \pm SEM. Statistical differences between different groups were evaluated using oneway ANOVA with the Tukey as post hoc test. The level of significance for all tests was set at $\mathrm{P}<0.05$.

\section{Results}

Our results showed that both training programs were increased transcript levels of MBP and PLP. The results indicated that IT program $(8.74 \pm 0.88, \mathrm{P}<0.05)$ had a greater stimulatory effect on the expression levels of MBP compared to CT group (6.95 \pm 1.1$)$ (fig 1). In addition, IT program $(5.42 \pm 0.48, \mathrm{P}<0.05)$ had a greater stimulatory effect on the expression levels of PLP compared to CT group (4.62 \pm 0.5 , $\mathrm{P}<0.032$ ) (Figure 2).

\section{Discussion}

Regular exercise improves the cognitive function of the brain, also promotes the plasticity of the brain. Considering the important role of myelin in physical and mental health, and nerve degeneration such as Alzheimer's disease, Parkinson's disease, multiple sclerosis 's disease, etc, (12) and there is not enough information about the effect of exercise intensity on myelination. Therefore, in this study, we investigated the effect of HIIT versus CT on myelin-synthesis related genes in the hippocampus of C57BL/6 mice. The results of this study showed that HIIT versus CT significantly improved the expression of MBP and PLP genes in the hippocampus of mice. It is likely that HIIT is more effective in neuronal protection in individuals than $\mathrm{CT}$ by improving the expression of myelin-related genes. The current findings are in agreement with other observations showing that exercise protects against demyelination (Kim et al., 2017; Lee et al., 2017; Bernardes et al., 2016; Yoon et al., 2016). For example, Kim and colleagues (2017) showed that treadmill training promotes the recovery of functions through the upregulation of myelin components of the brain and neurogenesis

(13). 


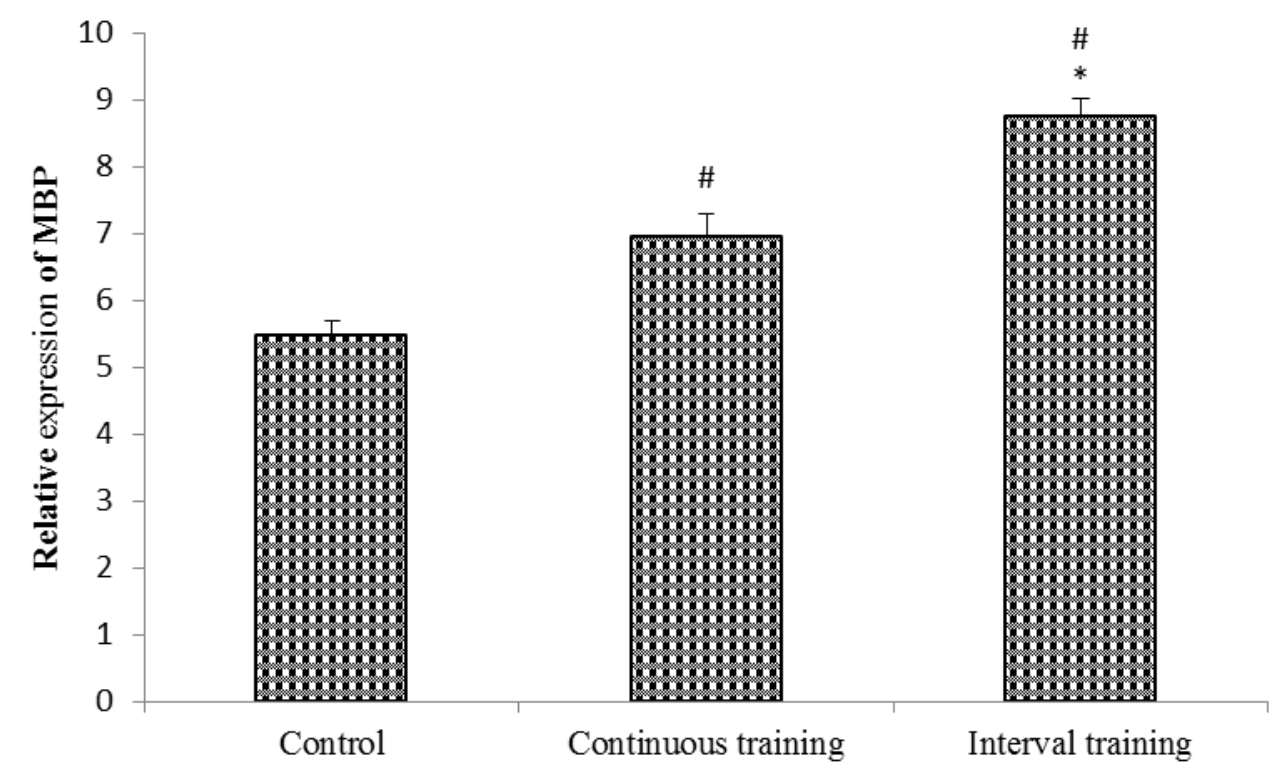

Figure1. Relative expression level of Myelin Basic Protein (MBP) (mRNA level) in hippocampus of different groups of rats. Data are means \pm SEM.

\#: Significantly different compared to control group $\mathrm{P}<0.05$

*: Significantly different compared to continuous training $\mathrm{P}<0.05$

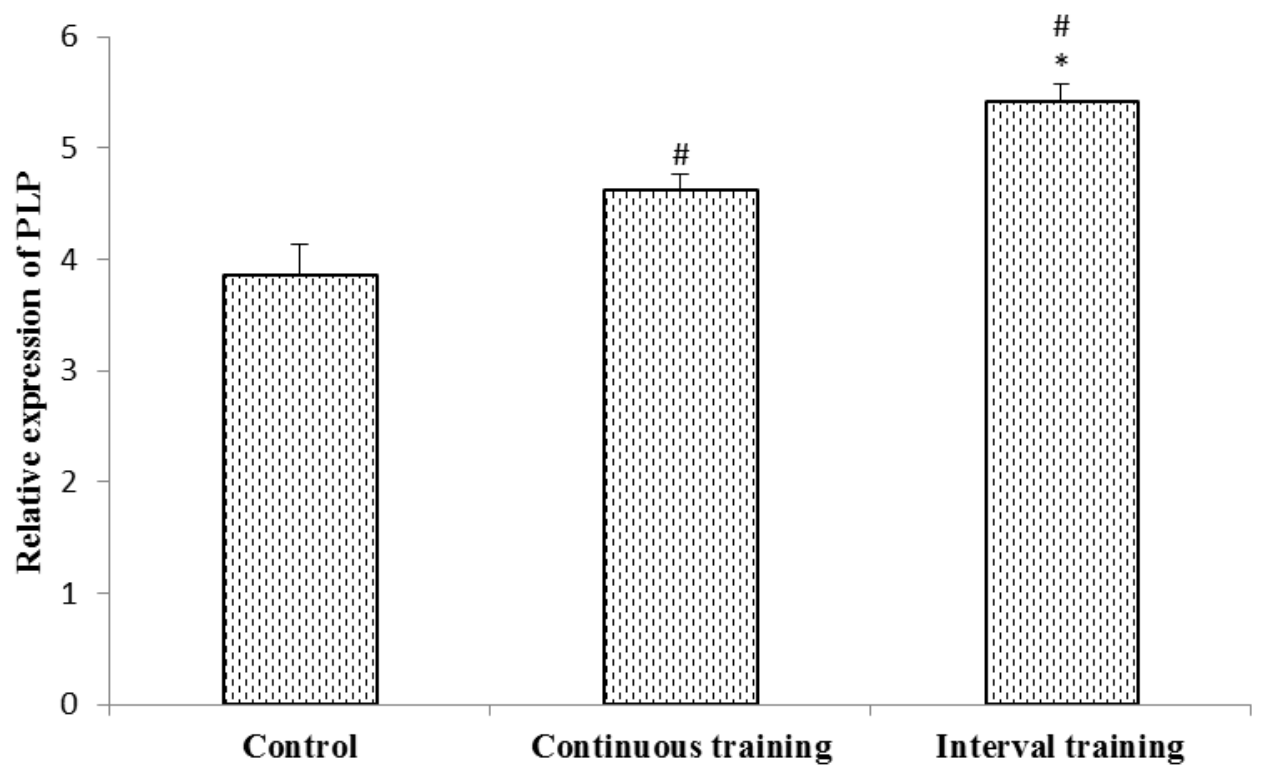

Figure 2. Relative expression level of proteolipid protein (PLP) (mRNA level) in hippocampus of different groups of rats. Data are means \pm SEM.

\#: Significantly different compared to control group, $\mathrm{P}<0.05$.

*: Significantly different compared to continuous training group, $\mathrm{P}<0.05$.

They showed that MBP is important for maintaining the structural stability of the myelin, and is an essential factor in the nerve conduction. Treadmill exercise can also prevent loss of MBP expression in the white matter after chronic cerebral hypoperfusion and provide protective effects on cognitive impairment and 
myelin damage (Lee et al., 2017) (14). High dietary fat and exercise training positively promote myelin protein expression (15).

Results of clinical exercise interventions have shown that exercise can reduce the axonal degeneration and improve neuronal protection in patients (16). It has been shown that exercise training play an endogenous neuroprotective role (17) and increase expression of neurotrophic factors in the hippocampus of mice and rats (18, 19). It also increased the proliferation of oligodendrocytes and improvement of myelination $(5,20)$. However, the effect of exercise on defense against disease largely depends on the volume and intensity of exercise $(21,22)$.

Zimmer et al. (2017) investigated the effect of two continuous and interval training programs for three weeks on cognitive function of MS patients (10). The difference between the results is probably due to short-term training periods or type of studied tissue. In the present study, brain tissue was examined but blood serum was studied in other studies.

So far, no study has been done to examine the direct effect of HIIT on myelination. And other studies that have examined the effects of HIIT on patients or healthy samples have focused on the characteristics of muscle contraction, antioxidant capacity, metabolic properties, neurotrophins and cognitive function, which resulted in a promotion of the physiological characteristics of HIIT.

In general, the results of this research and recent studies showed the positive effect of regular training on production of oligodendrocytes and myelin sheath formation $(5,23,24)$. Probably, HIIT may increase the proliferation, differentiation, maturation, and migration of neuronal cells rather than CT, and these cells are distinguished from other cell types with a more specialized function, such as neurons and glial cells. Thus, it is possible that
HIIT may be more effective to stimulate myelin formation than $\mathrm{CT}$ with increased velocity of differentiation and neurogenesis.

The basic mechanisms of neuroprotective effects of exercise are not yet fully understood, one of the possible mechanisms is reducing the formation of free radicals and the activity of antioxidant enzymes. In addition, HIIT can be considered as a type of tension in the brain, that causes changes such as neurogenesis, angiogenesis, and changes in the extracellular environment, they may help to brain adaptations to exercise.

In fact, recognizing the type of exercise training, according to its intensity and duration, helps to repair the damage from neurological disease, as well as the repair of synaptic communication. It is suggested that the effects of HIIT on antiinflammatory factors, oxidative stressors, and neuronal markers such as astrocytes, oligodendrocytes, and neurotrophins in neurological diseases should be investigated.

\section{Conclusion}

Overall, the results of the present study showed that the intensity of exercise training possibly improves myelination, this can lead to structural and functional changes in brain tissue against stress. In addition, the promoting of MBP and PLP levels following HIIT can indicate the response of nerve cells to intensely training. Therefore, further studies on the effect of HIIT on cellular and molecular changes in brain tissue are necessary.

\section{Acknowledgment}

This article is the result of Ph.D. thesis at Shahid Chamran University of Ahwaz. Therefore, we sincerely appreciate the personnel of the Faculty of Sport Science and Veterinary Medicine. 


\section{References}

1. Lindner M, Heine $\mathrm{S}$, Haastert $\mathrm{K}$, Garde $\mathrm{N}$, Fokuhl J, Linsmeier $\mathrm{F}$, et al. Sequential myelin protein expression during remyelination reveals fast and efficient repair after central nervous system demyelination. Neuropathol Appl Neurobiol. 2008; 34(1): 105-14. doi: 2990.2007.00879.x

$10.1111 / \mathrm{j} .1365-$

2. Quarles RH, Macklin WB, Morell P. Myelin formation, structure and biochemistry. In Basic neurochemistry: molecular, cellular and medical aspects. 7th edn (Siegel G. J., Albers R. W., Brady S. T. and Price D., eds.), Academic Press Elsevier, New York; 2006. 51-71.

3. Pagnini F, Bosma CM, Phillips D, Langer E. Symptom changes in multiple sclerosis following psychological interventions: a systematic review. BMC Neurol. 2014; 14(1): 222. doi.org/10.1186/s12883014-0222-z

4. Motl RW, Sandroff BM, Kwakkel G, Dalgas U, Feinstein A, Heesen C, et al. Exercise in patients with multiple sclerosis. Lancet Neurol. 2017; 16(10): 848-56. doi: 10.1016/S14744422(17)30281-8.

5. Bernardes $\mathrm{D}, \quad$ Brambilla $\mathrm{R}$, Bracchi-Ricard V, Karmally S, Dellarole A, Carvalho-Tavares J, et al. Prior regular exercise improves clinical outcome and reduces demyelination and axonal injury in experimental autoimmune encephalomyelitis. $\mathbf{J}$ Neurochem. 2016; 136: 63-73. doi: 10.1111/jnc.13354.

6. Patel DI, White LJ. Effect of 10-day forced treadmill training on neurotrophic factors in experimental autoimmune encephalomyelitis. Appl Physiol Nutr Metab. 2013; 38(2): 1949. doi: 10.1139/apnm-2012-0303.

7. Kim K, Shin MS, Cho HS, Kim YP. Effects of endurance exercise on expressions of glial fibrillary acidic protein and myelin basic protein in developing rats with maternal infection-induced cerebral palsy. J Exerc Rehabil. 2014;10(1):9-14. doi: 10.12965/jer.140084.

8. Naghibzadeh M, Ranjbar R, Tabandeh MR, Habibi A. Effects of two training programs on transcriptional levels of neurotrophins and glial cells population in hippocampus of experimental multiple sclerosis. Int $\mathbf{J}$ Sports Med. 2018; 39(8):604-12. doi: 10.1055/a-0608-4635.

9. Afzalpour ME, Chadorneshin HT, Foadoddini M, Eivari HA. Comparing interval and continuous exercise training regimens on neurotrophic factors in rat brain. Physiol Behav. 2015; 147: 78-83. doi: 10.1016/j.physbeh.2015.04.012.

10. Zimmer P, Bloch W, Schenk A, Oberste M, Riedel S, Kool J, et al. High-intensity interval exercise improves cognitive performance and reduces matrix metalloproteinases-2 serum levels in persons with multiple sclerosis: A randomized controlled trial. Mult Scler J. 2017: $1352458517728342 . \quad$ doi: $10.1177 / 1352458517728342$.

11. Pin-Barre C, Constans A, Brisswalter J, Pellegrino C, Laurin J. Effects of high-versus moderate-intensity training on neuroplasticity and functional recovery after focal ischemia. Stroke. 2017; 48(10): 2855-64. doi: 10.1161/STROKEAHA.117.017962.

12. Baek S-S. Role of exercise on the brain. J Exerc Rehabil. 2016; 12(5): 380-5. doi: 10.12965/jer.1632808.404

13. Kim HN, Pak ME, Shin MJ, Kim SY, Shin YB, Yun YJ, et al. Comparative analysis of the beneficial effects of treadmill training and electroacupuncture in a rat model of neonatal hypoxia-ischemia. Int $\mathrm{J}$ Mol Med. 2017; 39(6): 1393-402. doi: 10.3892/ijmm.2017.2970 
14. Lee JM, Park JM, Song MK, Oh YJ, Kim CJ, Kim YJ. The ameliorative effects of exercise on cognitive impairment and white matter injury from blood-brain barrier disruption induced by chronic cerebral hypoperfusion in adolescent rats. Neurosci Lett. 2017; 638: 83-9. doi: 10.1016/j.neulet.2016.12.018.

15. Yoon H, Kleven A, Paulsen A, Kleppe $\mathrm{L}, \mathrm{Wu} \mathrm{J}$, Ying $\mathrm{Z}$, et al. Interplay between exercise and dietary fat modulates myelinogenesis in the central nervous system. Biochim Biophys Acta. 2016; 1862(4): 545-55. doi: 10.1016/j.bbadis.2016.01.019.

16. White LJ, Castellano V. Exercise and Brain Health-Implications for Multiple Sclerosis. Sports Med. 2008; 38(2): 91-100. doi: 10.2165/00007256200838020-00001

17. Kim TW ,Sung YH. Regular exercise promotes memory function and enhances hippocampal neuroplasticity in experimental autoimmune encephalomyelitis mice. Neuroscience. 2017; 346: 173-81. doi: 10.1016/j.neuroscience.2017.01.016.

18. Cotman CW, Berchtold NC. Exercise: a behavioral intervention to enhance brain health and plasticity. Trends Neurosci. 2002; 25(6): 295-301. doi: 10.1016/S0166-2236(02)02143-4.

19. Gibbons TE, Pence BD, Petr G, Ossyra JM, Mach HC, Bhattacharya TK, et al. Voluntary wheel running, but not a diet containing (-)-Epigallocatechin-3- gallate and $\beta$-Alanine, improves learning ,memory and hippocampal neurogenesis in aged mice. Behav Brain Res. 2014; 272: 131-40. doi: 10.1016/j.bbr.2014.05.049.

20. Feter N, Freitas M, Gonzales N, Umpierre D, Cardoso R, Rombaldi A. Effects of physical exercise on myelin sheath regeneration: A systematic review and meta-analysis. Sci Sports. 2017; 33(1):8-21. doi: 10.1016/j.scispo.2017.06.009.

21. Pedersen BK, Hoffman-Goetz L. Exercise and the immune system: regulation, integration, and adaptation. Physiol Rev. 2000; 80(3): 1055-81. doi: 10.1152/physrev.2000.80.3.1055.

22. Terra R, Silva SAGd, Pinto VS, Dutra PML. Effect of exercise on immune system: response, adaptation and cell signaling. Rev Bras Med Esport. 2012; 18(3): 208-14. doi: 10.1590/S151786922012000300015.

23. Houdebine L, Gallelli CA, Rastelli M, Sampathkumar NK, Grenier J. Effect of physical exercise on brain and lipid metabolism in mouse models of multiple sclerosis. Chem Phys Lipids. 2017. doi: 10.1016/j.chemphyslip.2017.06.002.

24. Tomlinson L, Leiton CV, Colognato $\mathrm{H}$. Behavioral experiences as drivers of oligodendrocyte lineage dynamics and myelin plasticity. Neuropharmacology. 2016; 110: 548-62. doi: 10.1016/j.neuropharm.2015.09.016. 